\title{
Linha do cuidado: a emergência pediátrica na perspectiva da integralidade do cuidado
}

\author{
Line care: the pediatric emergency in wholeness perspective \\ care
}

\author{
Márcia Cristina de Almeida Cordovil Pires ${ }^{1} \bullet$ Simone Cruz Machado Ferreira² • Ana Lucia Abrahão da Silva ${ }^{3}$
}

\section{RESUMO}

Objetiva-se caracterizar a linha do cuidado que traduza a atenção prestada pela equipe multiprofissional em diferentes etapas da produção do cuidado na Emergência Pediátrica de um Hospital Universitário, na perspectiva da integralidade do cuidado através do uso da ferramenta “Fluxograma Analisador do Modelo de atenção".Apresentado como estudo qualitativo, cuja coleta de dados se deu por meio da observação sistemática no setor, acrescido da contribuição da equipe multiprofissional atuante através da técnica da vinheta. Resultou-se a construção da linha de cuidado que traduz a atenção prestada pela equipe multiprofissional na Emergência Pediátrica. Conclui-se que a construção do fluxograma permitiu identificar os principais processos de trabalho, bem como avaliar uma série de atividades que implicam em novos processos em outras áreas da organização evidenciando, assim, o trabalho interdisciplinar. Contribuindo não só para o atendimento seguro e de qualidade, mas também favorecendo o ensino e a pesquisa desenvolvida neste ambiente do Hospital.

Palavras-chave: Sistemas de Saúde; Assistência Integral a Saúde; Gestão em Saúde; Enfermagem Pediátrica; Enfermagem.

\begin{abstract}
The aim is to characterize the care line that reflects in the attention provided by the multidisciplinary team at different stages of production in the Pediatric Emergency Care in a University Hospital from the perspective of comprehensive care through the use of the tool "Flowchart attention Model Analyzer". Presented as a qualitative study, whose data collection was through systematic observation of the "day to day" in the industry, plus the multi-professional team of the contribution that there acts by the vignette technique. Resulting in a care line construction that reflects the attention paid by the multidisciplinary team at the Pediatric Emergency. It concludes that the construction of the flow chart has identified the main work processes and evaluate a range of activities that include new processes in other areas of the organization thus highlighting the interdisciplinary work. Contributing not only to safe and quality care, but also promoting the teaching and research conducted in this hospital environment.
\end{abstract}

Keywords: Health Systems; Integral Assistance to Health; Health Management; Pediatric Nursing; Nursing.

Os autores declaram não haver conflitos de interesse nem fontes de financiamento.

${ }^{1}$ Enfermeira. Mestranda do Mestrado Profissional Enfermagem Assistencial (MPEA) da EEAAC/UFF. Coordenadora de Enfermagem da Emergência Pediátrica do HUAP/UFF. Especialista em Administração dos Serviços de Enfermagem da EEAAC/UFF. Membro do Núcleo de Estudos e Pesquisas em Cidadania e Gerência na Enfermagem (NECIGEN). E-mail: mcacpires@hotmail.com. Este artigo faz parte da dissertação de mestrado em desenvolvimento intitulada "Banco de Dados de Emergência Pediátrica do Hospital Universitário Antônio Pedro: Ferramenta Gerencial".

${ }^{2}$ Enfermeira. Doutora em Enfermagem. Professora Associada III do Departamento de Fundamentos de Enfermagem e Administração da Escola de Enfermagem Aurora de Afonso Costa (EEAAC) da Universidade Federal Fluminense (UFF). Docente Permanente do Mestrado Profissional Enfermagem Assistencial (MPEA) da EEAAC/UFF. Membro do Núcleo de Estudos e Pesquisas em Cidadania e Gerência na Enfermagem (NECIGEN).E-mail: s.cruz.ferreira@uol.com.br

${ }^{3}$ Enfermeira. Doutora em Saúde Coletiva na área de Gestão e Planejamento em Saúde. - Diretora da EEAAC-UFF. E-mail:abrahaoana@gmail.com 


\section{INTRODUÇÃO}

O atendimento no campo da saúde à criança é caracterizado por peculiaridades biológicas e psicológicas próprias desse grupo populacional, sujeito aos agravos decorrentes das doenças prevalentes na infância, como também aos agravos entendidos como situações de emergência, por exemplo, traumas e envenenamentos, necessitando de recursos materiais e humanos especializados ${ }^{1}$.

É possível afirmar que, na emergência pediátrica, podem ocorrer situações graves e ameaçadoras da vida da criança, pressionada pelo tempo, em que cada segundo pode fazer a diferença. Além disso, acrescenta-se nesse contexto assistencial a participação dos familiares, uma vez que os pais estão incorporados ao atendimento e, portanto, fazem parte da rede do cuidado que, ao mesmo tempo em que relatam as intercorrências com a criança que os levaram a procurar o serviço de emergência, também presenciam as intervenções que podem ser bem sucedidas ou não.

No setor de urgências de qualquer hospital, o enfermeiro é um elemento central nas várias áreas da prestação do socorro, desde a triagem à reanimação, à cirurgia ou trauma ou à sala de observações, entre outras. Em cada uma dessas áreas, espera-se que, nas atividades autônomas como nas interdependentes, o enfermeiro atue de forma segura, respeitando os direitos dos doentes e com base em evidências científicas. Mas a atuação em contexto de urgência é uma área do saber de vastas proporções que compreende tanto procedimento simples como de grande complexidade 2 .

Assim, torna-se possível entender a proposta deste estudo que se refere à inserção da Emergência Pediátrica do Hospital Universitário Antônio Pedro na rede de assistência à saúde, tomando como premissa a linha da integralidade do cuidado. 0 interesse por esse tema emergiu no cotidiano de trabalho na emergência pediátrica e da necessidade de se reestruturar as ações de enfermagem nesse setor a fim de adequá-las às novas exigências do Sistema Único de Saúde (SUS).

A primeira tarefa, quando se pensa no tema da integralidade da atenção no ambiente hospitalar, é, sem dúvidas, estabelecermos uma definição de integralidade que sirva de guia para a condução de nossas reflexões. Poderíamos dizer, então, que atenção integral de um paciente no hospital seria o esforço de uma abordagem completa, holística, portanto, integral, de cada pessoa portadora de necessidades de saúde que, por um certo período de sua vida, precise de cuidados hospitalares. Tal abordagem implicaria garantir desde o consumo de todas as tecnologias de saúde disponíveis para melhorar e prolongar a vida, até a criação de um ambiente que resultasse em conforto e segurança para a pessoa hospitalizada ${ }^{3}$.

O cuidado integral em saúde ocorreria a partir de uma combinação generosa e flexível de tecnologias duras, leve duras e leves, em que entendemos por tecnologias duras aquelas ligadas a equipamentos e procedimentos; tecnologias leve duras aquelas decorrentes do uso de saberes bem estruturados, como a clínica e a epidemiologia; e tecnologias leves como aquelas relacionadas ao espaço intersubjetivo do profissional de saúde e do paciente 4 .

As ações de enfermagem prestadas à criança e sua família atendidas na Emergência Pediátrica do Hospital Universitário Antônio Pedro, a partir da compreensão de sua inserção na rede de assistência à saúde, estão articuladas, não só ao emprego das tecnologias do trabalho em saúde, mas também à lógica da integralidade voltada à gestão do serviço no que se refere à tomada de decisões baseada em informações reais que poderão gerar mudanças e melhorias no planejamento da assistência, investindo, assim, no desenvolvimento/aprimoramento de estratégias para potencializar a qualidade do cuidado prestado e as condições de trabalho das equipes de saúde.

Diante disso, o objeto desse estudo é a construção da linha do cuidado na Emergência Pediátrica estabelecendo suas articulações com o cuidado integral à saúde, definindose linha do cuidado como uma estratégia de organização da atenção que viabiliza a integralidade da assistência por meio de um conjunto de saberes, tecnologias e recursos necessários ao enfrentamento de riscos, agravos ou demais condições específicas do ciclo de vida ou outro critério sanitário a serem ofertados de forma oportuna, articulada e contínua, abrangendo os campos de promoção, prevenção, tratamento e reabilitação ${ }^{5}$.

Desta forma, formulou-se como objetivo: caracterizar a linha do cuidado que traduza a atenção prestada na Emergência Pediátrica do Hospital Universitário Antônio Pedro na perspectiva da integralidade do cuidado.

Neste movimento de reflexão, entendemos a linha do cuidado como a imagem pensada para expressar os fluxos assistenciais seguros e garantidos ao usuário, no sentido de atender às suas necessidades de saúde. A linha do cuidado tem seu início na entrada do usuário por qualquer via do sistema de saúde que envolve assistência, seja a visita domiciliar, urgência, ambulatório, equipe de saúde na atenção básica, ou qualquer ponto onde ocorre interação entre profissional de saúde e usuário ${ }^{6}$.

\section{MÉTODO}

Trata-se de um estudo descritivo-exploratório acerca da inserção do atendimento da Emergência Pediátrica do Hospital Universitário Antônio Pedro na rede de assistência à saúde, que utilizou a abordagem qualitativa na análise dos dados coletados. Essa abordagem responde a questões muito particulares, pois se preocupa, nas ciências sociais, com um nível de realidade que não pode ser quantificado. Ou seja, ela trabalha com o universo de significados, motivos, aspirações, crenças, valores e atitudes, o que corresponde a um espaço mais profundo das relações, dos processos e dos fenômenos que não podem ser reduzidos à operacionalização de variáveis ${ }^{7}$. 
0 estudo exploratório permite ao investigador aumentar sua experiência em torno de um determinado problema e visa maior familiaridade em relação a um fato ou fenômeno. Também, em se tratando de estudo descritivo, caracteriza-se pela necessidade de se explorar uma situação não conhecida, da qual se tem necessidade de maiores informações ${ }^{8}$.

O cenário do estudo é a Emergência Pediátrica do Hospital Universitário Antônio Pedro que é uma instituição vinculada à Universidade Federal Fluminense (UFF), situada no município de Niterói no estado do Rio de Janeiro, com atividades de ensino, pesquisa e assistência, que atende, exclusivamente, pacientes do SUS. É referência para região metropolitana 2 que abrange os municípios de Niterói, São Gonçalo, Itaboraí, Rio Bonito, Maricá, Tanguá e Silva Jardim.

Foram sujeitos da pesquisa a equipe multidisciplinar atuante na Emergência Pediátrica no período de janeiro a maio de 2015, sendo excluídos os servidores que estivessem de férias e/ou licença médica.

Após aprovação no Comitê de Ética CCM/HUAP, com o CAAE $n^{\circ} 39142714.8 .0000 .5243$ e parecer de aprovação $n^{\circ}$ 924.317de 04/12/2014, foi iniciada a coleta de dados. Por tratar-se de uma dissertação de mestrado em desenvolvimento, este estudo apresenta os resultados relacionados ao alcance de um dos objetivos da pesquisa intitulada "Banco de Dados de Emergência Pediátrica do Hospital Universitário Antônio Pedro: Ferramenta Gerencial". Os dados foram coletados através de observação sistemática direta, realizados no período de janeiro a maio de 2015 , totalizando 60 horas.

Os dados que emergiram das observações e sua articulação aos conceitos relacionados à linha do cuidado fundamentados, principalmente, nos estudos de Merhy, utilizando como ferramenta o Fluxograma Analisador do Modelo de Atenção do Serviço de Saúde (1997), subsidiaram a caracterização da linha do cuidado que traduz a atenção prestada na Emergência Pediátrica do Hospital Universitário Antônio Pedro na perspectiva da integralidade do cuidado.

Também, foi realizada a técnica da vinheta que consiste em descrições breves de eventos ou situações das quais os respondentes são solicitados a reagir. As descrições podem ser fictícias ou reais, mas sempre são estruturadas de modo a explicar informações sobre as percepções, opiniões ou conhecimentos dos respondentes sobre algum fenômeno estudado ${ }^{9}$. A técnica da vinheta permitiu que a linha de cuidado elaborada fosse analisada a partir da percepção dos profissionais que desenvolvem seus processos de trabalho no bojo do projeto terapêutico representado nessa linha.

A construção do fluxograma obedeceu a dois eixos centrais a observação do cotidiano do trabalho na Emergência e o material produzido com a técnica da vinheta, junto aos profissionais de saúde.

\section{RESULTADOS}

O fluxograma elaborado na figura 1 , explicita as etapas do processo de trabalho que são representadas por meio de símbolos convencionados, onde a elipse representa a entrada ou saída do processo; o losango indica os momentos em que há discussões importantes para continuidade da assistência e, por fim, o retângulo se relaciona ao momento de uma ação/intervenção ${ }^{10}$.

O fluxograma é um diagrama muito utilizado com o objetivo de desenhar como está organizado um conjunto de processos de trabalho que se vinculam entre si em torno de determinada cadeia de produção. Isto é, ele é uma representação gráica, que busca a partir do processo de trabalho, apreender tanto os caminhos percorridos pelo usuário como a sua inserção no interior do serviço de saúde, quando procura por cuidado. Esta ferramenta possibilita aguçar o olhar sobre os fluxos existentes no momento da produção do cuidado em saúde identificando os seus problemas. Sua aplicação lança luz a áreas de sombra que operam no sentido contrário a um cuidado integral e que tem sua centralidade no usuário e em suas necessidades ${ }^{11}$.

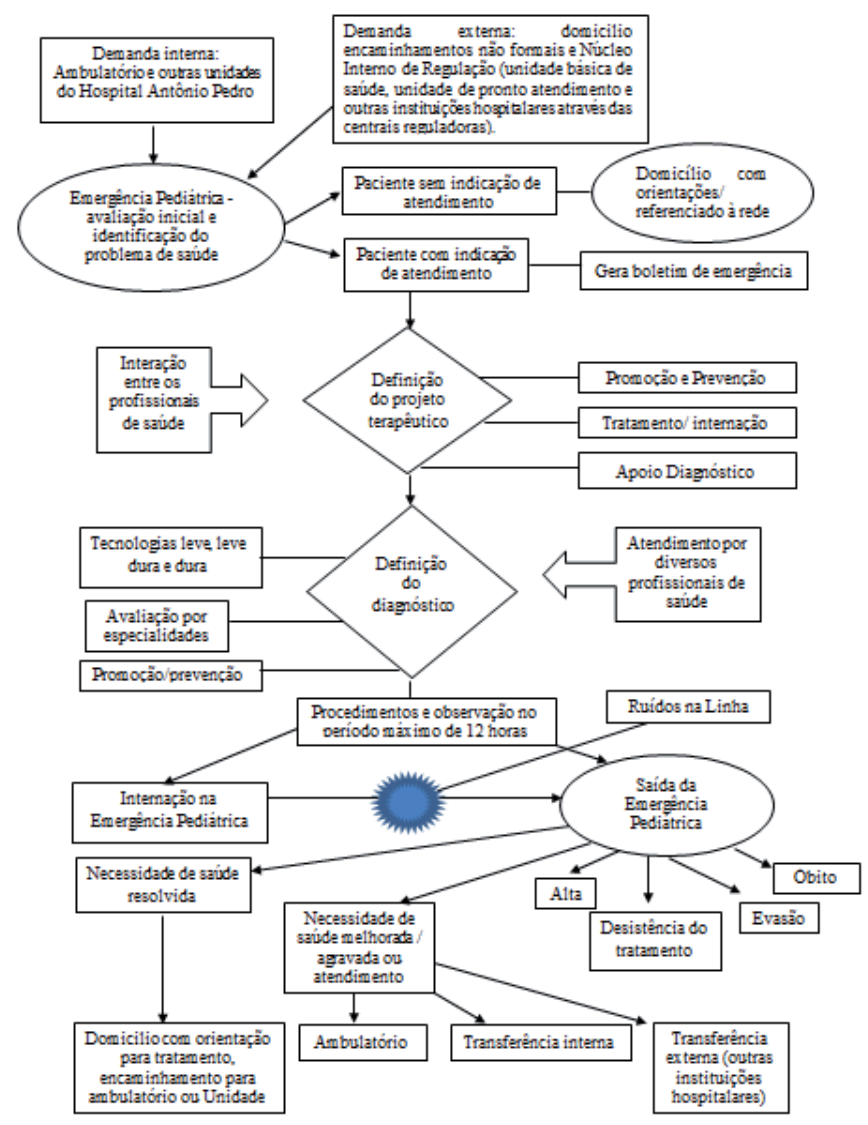

Figura 1: Representação da linha do cuidado na Emergência Pediátrica do Hospital Universitário Antônio Pedro.

Fonte: elaboração própria.

\section{DISCUSSÃO}

Nas organizações de saúde em geral e no hospital em particular, o cuidado é, por sua natureza, necessariamente multidisciplinar. Isto é, depende da união do trabalho 
de vários profissionais. 0 cuidado, de forma idealizada, recebido/vivido pelo paciente é somatório de um grande número de pequenos cuidados parciais que vão se complementando, de maneira mais ou menos consciente e negociada, entre os vários cuidadores que circulam e produzem a vida do hospital, incluindo o usuário. Assim, vai se formando um complexo conjunto que se complementa de atos, de procedimentos, de fluxos, de rotinas, de saberes que compõem o que entendemos como cuidado em saúde. A maior ou menor integralidade da atenção recebida, na prestação desse cuidado, depende da forma como se articulam as práticas dos trabalhadores em saúde inseridos nesse processo ${ }^{3}$.

Neste movimento de reflexão, através da observação direta do cotidiano do processo de trabalho da equipe multidisciplinar atuante na Emergência Pediátrica do Hospital Universitário Antônio Pedro, podemos constatar que a organização desse trabalho da equipe de saúde apresenta momentos diferenciados: a recepção e acolhimento, 0 atendimento as urgências e emergências, a assistência aos pacientes internados e/ou em observação e a atenção aos usuários com demandas de necessidades de saúde não urgente.

A criança e sua família chegam à unidade de Emergência Pediátrica do Hospital Universitário Antônio Pedro por três caminhos distintos: oriundas de unidades básicas de saúde, unidades de pronto atendimento e outras instituições hospitalares através das centrais reguladoras; encaminhadas pelo ambulatório e outras unidades do Hospital Universitário Antônio Pedro, e, por fim, ocorre a demanda espontânea, entendida aqui como crianças oriundas de suas residências ou outras localidades.

Ressalta-se que o modelo assistencial idealizado pela população ainda é a atenção médica hospitalar, onde o hospital, representado pelo serviço de emergência, aparece como local que reúne um somatório de recursos para o atendimento, quais sejam: consultas médicas, procedimentos de enfermagem, medicamentos, exames laboratoriais e de imagem e internações. Notadamente, é possível destacar a prevalência da visão do atendimento à saúde com enfoque na dimensão biológica.

0 regulamento técnico dos sistemas de urgência e emergência preconiza que os serviços de emergência sejam providos de acolhimento com triagem classificatória de risco e atendimento subsequente, conforme a necessidade do caso. Em situação de demanda inadequada, o regulamento preconiza que ocorra o redirecionamento do paciente para a devida inserção no sistema de atenção à saúde ou para prosseguimento do tratamento ${ }^{12}$.

0 acolhimento é uma ação tecno-assistencial que visa atender todos os que procuram os serviços, acolhendo, escutando e pactuando respostas adequadas aos usuários. Já a tecnologia de avaliação com classificação de risco, segundo o Ministério da Saúde, pressupõe a determinação de agilidade no atendimento a partir da análise, sob a ótica de protocolo pré-estabelecido, do grau de necessidade do usuário, proporcionando a atenção centrada no nível de complexidade e não na ordem de chegada ${ }^{13}$. A grande crise que envolve redução de recursos humanos pelo qual o Hospital Universitário Antônio Pedro vem passando, levou a desativação do grupo de acolhimento, pois seus componentes foram realocados em outras unidades de atenção. Por isso, quando a criança e sua família chegam à Emergência Pediátrica, elas são acolhidas primeiramente pela equipe de enfermagem lá atuante, que, através da escuta, identifica o problema de saúde e solicita a presença do médico pediatra que fará uma avaliação inicial da demanda, o que poderá gerar uma consulta médica ou não. Caso a criança não necessite do atendimento na emergência, ela e sua família serão referenciadas à rede ou ao domicílio com orientações para saúde. No caso da necessidade da consulta médica, a família será orientada para abertura do boletim de emergência. A partir dessa decisão é definido um "projeto terapêutico", que inclui diagnóstico, tratamento/internação, promoção e prevenção para o atendimento com a participação dos profissionais de saúde atuantes na unidade.

A linha do cuidado funciona com base nos projetos terapêuticos, aqui entendidos como um conjunto de atos assistenciais pensados para resolver determinado problema de saúde do usuário, com base em uma avaliação de risco. Ele ganha materialidade se for executado através da ação do trabalho sobre o usuário "portador de problema de saúde" e, isto ocorrendo, deixa de ser "projeto" para se transformar em atos concretos assistenciais ${ }^{3}$.

Acolhimento, vínculo e responsabilização são diretrizes da linha do cuidado, pois para cuidar de fato do usuário é necessário que os serviços de saúde organizem seus processos de trabalho de modo que haja o acolhimento dos usuários pelos trabalhadores, o que significa atender bem, fazer uma escuta qualificada do seu problema de saúde, resolver se necessário, fazer um encaminhamento seguro, e isto só é possível se esta rede estiver operando com base na linha do cuidado ${ }^{4}$.

Os pacientes com potencial risco de vida têm sempre seu atendimento priorizado. Neste momento observase que os profissionais da equipe de saúde do setor organizam suas ações para o restabelecimento da vida, o que remete ao trabalho em equipe e a articulação entre as diferentes categorias profissionais no atendimento às situações de urgência e emergência. Esse achado corrobora os resultados de um estudo que destaca a importância da integração e articulação das diferentes categorias profissionais e ramos do conhecimento em prol de uma assistência oportuna e livre de riscos, pautada na troca, cooperação e respostas imediatas ao usuário nas unidades de emergências hospitalares ${ }^{7}$.

Com a definição do diagnóstico e a confirmação do problema de saúde, a criança e sua família passarão a receber o atendimento de vários profissionais de saúde; sendo avaliada por especialistas, terá à sua disposição as tecnologias disponíveis na unidade hospitalar e/ 
ou em outras unidades, caso seja necessário. Poderá ser submetida a determinados procedimentos, por exemplo: coleta de material biológico para exames, exames de imagem, medicação e observação por um período máximo de 12 horas. Ao término desse período, cessa o atendimento na emergência. Caso a criança tenha a sua necessidade de saúde atendida, ela receberá alta hospitalar e será encaminhada ao domicilio com orientações de promoção e prevenção, bem como será referenciada à rede básica ou ao ambulatório de especialidades do Hospital Universitário Antônio Pedro, através da solicitação de "continuidade de atenção" feita pelo médico responsável pelo seu atendimento na Emergência Pediátrica.

No entanto, se a necessidade de saúde não foi resolvida no período de 12 horas ela deverá ser internada nas unidades especializadas do hospital. Não havendo vagas nessas unidades, ela deverá ser internada na emergência pediátrica até que aconteça a vaga. Caso o Hospital Universitário Antônio Pedro não possua a especialidade que a criança necessite, a mesma terá vaga solicitada pelo plantonista médico em outras unidades de saúde, através da central reguladora, a fim de assegurar que sua saúde seja restabelecida na integralidade para que possa continuar seu ciclo de desenvolvimento.

Cabe destacar ainda que, além da atenção prestada às crianças que permanecem internadas em observação e os atendimentos em que o risco de vida é eminente, a equipe de saúde atuante na Emergência Pediátrica dividese ainda na atenção aos pacientes que necessitam de consulta médica, procedimento de enfermagem, exames e medicações. Essa demanda é constituída por pacientes que buscam na Emergência Pediátrica a solução para os mais diversos problemas sociais e de saúde. Essa diversidade de tarefas gera sobrecarga de trabalho entre os profissionais, visto que é complexo realizar múltiplas atividades ao mesmo tempo, especialmente, tratando-se de cuidado prestado a clientela infantil estando ela em estado grave ou não.

Pesquisa realizada em um serviço público de urgência pediátrica evidenciou a heterogeneidade dos atendimentos contrariando a vocação do serviço idealizado como referência para casos agudos. Os médicos ressentiam-se de assistir crianças que deveriam estar sendo atendidas no cuidado primário, expressando preocupação com as mais graves, que também chegavam necessitando de cuidados que não estão disponíveis em outros níveis do sistema ${ }^{14}$.

Torna-se importante compreender e salientar que o significado de urgência para os profissionais e para a população são distintos, como também são diferentes os conceitos de doenças e saúde nas suas definições biomédicas e nas representações e práticas, que variam segundo segmentos e culturas da sociedade. Para os profissionais, as urgências estão relacionadas às patologias que comprometem a vida ou função vital importante. Para a população, relacionam-se a necessidades variadas, incluindo aflição, angústia, abandono e miséria, que requerem ajuda e ou assistência, com solução imediata a uma dificuldade passageira ${ }^{15}$

Devido à complexidade das relações que acontece nos processos de trabalho em serviços hospitalares de emergência, o Ministério da Saúde enuncia o Fluxograma Analisador como uma importante ferramenta teórica para reflexão sobre a atuação da equipe na operacionalização do trabalho e na construção da relação de acolhimento do usuário ${ }^{6}$.

Nessa linha de raciocínio, podemos considerar o Fluxograma Analisador como instrumento potente que proporciona autoanálise e autogestão ao identificar as fragilidades e os interesses envolvidos na distribuição e na organização do processo de trabalho na Emergência Pediátrica do Hospital Universitário Antônio Pedro.

\section{CONCLUSÃO}

Ao se falar em qualidade de atendimento em serviço hospitalar de emergência, é necessário que aconteçam ações em todos os níveis hierárquicos, de seus trabalhadores, para que o resultado não seja nada menos que a resolução dos agravos de saúde em seu devido tempo e local. Aponta-se, portanto, a necessidade de organização do processo de trabalho na Emergência Pediátrica do Hospital Universitário Antônio Pedro com base nos pressupostos da Política Nacional de Atenção às Urgências e Emergências que preconizam o acesso e acolhimento aos serviços de saúde de acordo com sua complexidade tecnológica, reordenando, quando necessário, os serviços ambulatoriais básicos ou especializados existentes na rede de atenção à saúde. Consequentemente, é necessário conhecer a estrutura dos serviços e estabelecer a rede de atenção às urgências, com grades de referência e contra referência efetivamente pactuadas, de modo a corrigir as distorções ainda existentes nas portas de entrada do sistema de saúde.

A trajetória percorrida neste estudo permitiu identificar os principais processos de trabalho, bem como descrever os fluxos de um serviço de emergência pediátrica e analisar uma série de atividades que implicam novos processos em outras áreas da organização como: recepção, enfermagem, equipe médica, laboratórios, serviço social, farmácia, nutrição, psicologia, higienização e área administrativa em geral, evidenciando, assim, as integrações para o bom atendimento à criança e sua família. Possibilitou também visualizar que a construção de uma linha do cuidado para a prestação de assistência no âmbito hospitalar auxilia na organização interna dos fluxos e processos de trabalho de um serviço de emergência e urgência, facilitando o dimensionamento de profissionais, a distribuição de materiais e medicamentos, como também outros aspectos relacionados à estrutura do serviço. Contribuindo não só para o atendimento seguro e de qualidade, mas também favorecendo o ensino e a pesquisa desenvolvidos nesse ambiente do hospital universitário. 


\section{REFERÊNCIAS}

1. Lima LMB, Almeida NMGS. Procura da emergência pediátrica pelas mães: implicações para superlotação. Saúde em Debate Rio de Janeiro. [Internet] 2013 [acesso em 30 abril 2016] Jan-Mar, 37(96):51-61. Disponível em: http://www. scielo.br/pdf/sdeb/v37n96/07.pdf

2. Malagutti W, Caetano KC. Gestão do Serviço de Enfermagem no Mundo Globalizado. Rio de Janeiro: Editora Rubio; 2009.

3. Cecilio LCO, Merhy EE. Integralidade do cuidado como eixo da gestão hospitalar. In: Pinheiro R, Mattos RA. Construção da integralidade: cotidiano saberes e práticas em saúde. Rio de Janeiro: IMS ABRASCO, 2003, p. 197-210.

4. Merhy EE. Saúde: a cartografia do trabalho vivo. São Paulo: HUCITEC 2002.

5. Portaria $n^{\circ} 3.390$, de 30 de dezembro de 2013 que institui a Política Nacional de Atenção Hospitalar (PNHOSP) no âmbito do Sistema Único de Saúde (SUS), estabelecendo-se as diretrizes para a organização do componente hospitalar da atenção à saúde (RAS).

6. Malta DC, Merhy EE. O percurso da linha do cuidado sob perspectiva das doenças crônicas não transmissíveis. Rev. Interface-comunicação, Saúde e Educação. [Internet] 2010 [acesso em 30 maio 2016] Jul-Set, 14(34):593-606. Disponível em: http://www.scielo.br/pdf/icse/v14n34/ aop0510.pdf

7. Alves M, Ramos FRS, Penna CMM. O trabalho interdisciplinar - aproximações possíveis na visão de enfermeiros de uma unidade de emergência. Rev. Eletrônica Texto Contexto Enferm. [Internet]. 2005 [acesso em 30 maio 2016] Jul-Set, 14(3):323-31. Disponível em: http://www.scielo.br/pdf/ tce/v14n3/v14n3a02.pdf

8. Peduzzi M. Equipe multiprofissional de saúde: conceito e tipologia. Rev. Saúde Pública [Internet]. 2001 [acesso em 23 maio 2016] 35(1):103-109 ISSN 1518-8787. Disponível em:http://www.uff.br/tcs2/images/stories/Arquivos/ textos_4p/trabalho_em_equipe/Peduzzi_2001.pdf

9. Polit DF, Hungler BP. Fundamentos da pesquisa em enfermagem. $7^{\mathrm{a}}$ ed. Porto Alegre, Artmed, 2011.

10. Franco TB, Merhy EE. $O$ uso de ferramentas analisadoras para apoio ao planejamento dos serviços de saúde: o caso do serviço social do hospital das clinicas da Unicamp. In: Merhy EE et al. Trabalho em saúde: olhando e experimentando o SUS no cotidiano. $4^{\mathrm{a} e d .}$ São Paulo: Editora Hucitec, 2007.

11. Merhy EE. Em busca do tempo perdido: a micropolítica do trabalho vivo em saúde, In: Merhy EE e Onoko R (orgs). Agir em saúde; um desafio para o publico. São Paulo: HUCITEC, 1997, p. 71-112.

12. Brasil. Ministério da Saúde. Portaria $n^{\circ} 1.600,7$ de julho de 2011, reformula a Política Nacional de Atenção às Urgências e institui a Rede de Atenção as Urgências no Sistema Único de Saúde (SUS).

13. Brasil. Ministério da Saúde. Secretaria Executiva. Núcleo Técnico de Política Nacional de Humanização. Humaniza SUS: Política Nacional de Humanização como eixo das praticas de atenção e gestão em todas as instancias do SUS/ Ministério da Saúde. Secretaria Executiva. Núcleo da Política Nacional de Humanização - Brasília: Ministério da Saúde 2004.
14. Melo EMC, Assunção AA, Ferreira RA. O trabalho dos pediatras em um serviço publico de urgência: fatores intervenientes no atendimento. Cad. Saúde Pública [Internet] 2007 [acesso em 20 março 2016] dez; 23(21): 3000-10. Disponível em: http://www.scielosp.org/pdf/csp/v23n12/20.pdf

15. Martinez AM, Nitschke CAS, organizadores. Regulação médica dos serviços de atendimento médico de emergência: SAMU 1999. 